\title{
Histology of Muscle Development in Pigs, Epigenetics from Myotubes to Tapered Fibres
}

\author{
Howard J. Swatland \\ University of Guelph, \\ Guelph, Canada \\ Email: Swatland [AT] uoguelph.ca
}

\begin{abstract}
Pre-natal muscle development in pigs starts with myotubes (axial nuclei in a tube of myofibrils) and secondary fibres (peripheral nuclei on an axial strand of myofibrils). By the time of birth, the nuclei of myotubes move to a peripheral position like secondary fibres. As pre-natal secondary fibres grow in length, the number of fibres in a transverse section may appear to increase. This stereology may also occur in post-natal muscles that have tapered fibres anchored in endomysial connective tissue around adjacent fibres and with one or both ends not reaching the end of their fasciculus. Up to 100 days gestation, Peroneus longus (no tapered fibres) had larger $(P<0.001)$ diameter secondary fibres than Longissimus thoracis (with tapered fibres). Up to 100 days gestation, no radial growth of secondary fibres was detected, but myotubes decreased in diameter $(P<0.001)$. From a curve showing the relative numbers of myotubes and secondary fibres, it was deduced that approximately $80 \%$ of muscle fibres in pigs are derived from secondary fibres. In post-natal Sartorius muscle there was an increase $(P<0.005)$ in the apparent number of muscle fibres attributed to longitudinal growth of tapered fibres. Myotubes located centrally within their fasciculi had the same position as slow-contracting fibres with a high myoglobin content in adult muscle. Post-natal changes in muscle fibre histochemistry were achieved through transitional types, probably neurally regulated rather than by differential longitudinal growth of tapered endings. Secondary fibres are important - they give rise to both the majority of muscle fibres in adult pigs and affect subsurface optical pathways and pork colourimetry.
\end{abstract}

Keywords - Pig muscle growth, Myotubes, Tapered fibres, Light scattering in pork

\section{INTRODUCTION}

Classical studies on the histology of skeletal muscle in pigs start with Theodor Schwann (1910-1882) who discovered that the skeletal muscle fibres of pigs may start in either of two ways, either with myoblasts fusing to form myotubes with axial nuclei within a tube of myofibrils, or with myoblasts aligning to form secondary fibres on an axial strand of myofibrils (Fig. 1, [1]). Then John MacCallum (1876-1906) showed that, as secondary fibres grew in length in the pre-natal pig, they increased the number of fibres seen in a cross section of a muscle (Fig. 2, [2]). Very few muscles have all their fibres running from origin to insertion, and many adult muscles may have some tapered fibres attached in the endomysial connective tissue of adjacent fibres. If tapered fibres grow in length faster than their fasciculi, the number of fibres appearing in a transverse section may increase without any new fibres being formed.

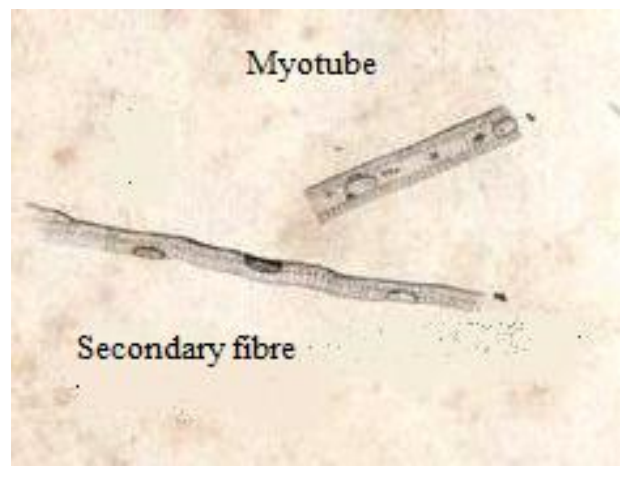

Fig. 1. Schwann's discovery that foetal pigs have two types of muscle fibres. Myotubes have axial nuclei within of a tube of myofibrils. Secondary fibres have an axial strand of myofibrils on which are located the nuclei (from Fig. 3 of Schwann [1]). 

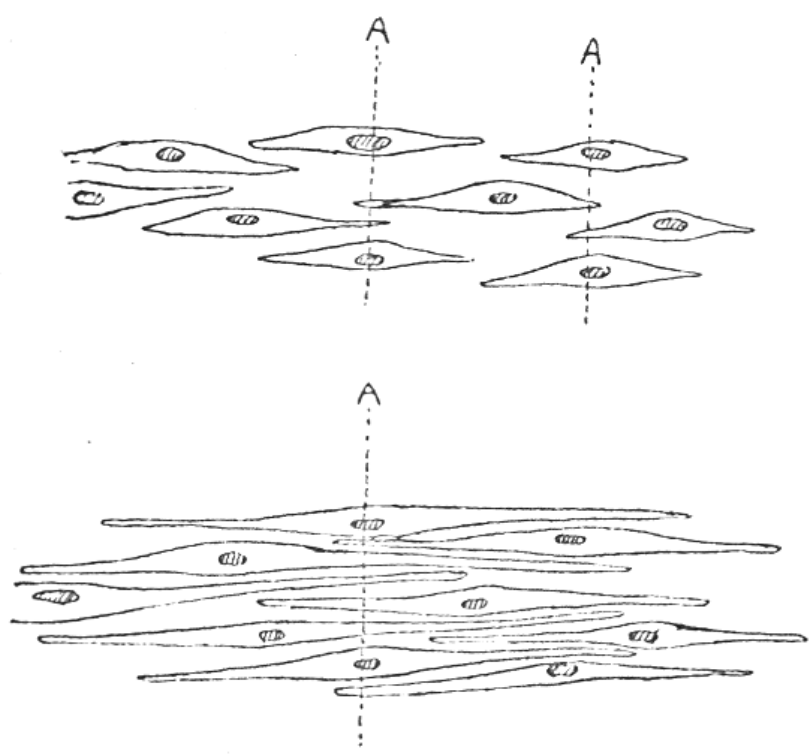

(2)

(2)
I

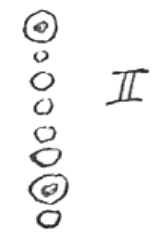

Fig. 2. MacCallum's stereology showing how growth in fibre length may change the apparent number of muscle fibres in a transverse section (from Fig. 6 of MacCallum [2]).

As a summary of what is known now about pre-natal development of muscle in pigs, mitotic division of premyoblasts creates myoblasts that link their cytoplasmic arms to fuse into the ends of myotubes. Myotubes have a tube of myofibrils surrounding axial nuclei. Later forming myoblasts join up with an axial core of myofibrils on the surfaces of primary myotubes to form secondary fibres. Myotubes that have managed to reach a muscle origin and insertion can now contract, thus throwing their attached secondary fibres into sinuous folds that cause their detachment from the primary myotube. This creates free space for a new generation of secondary fibres to form on the primary myotube. Peripheral axons grow into the muscle to innervate myotubes. Functional neuromuscular junctions are developed, and contractions cause axial nuclei to move to a peripheral position. Surviving myoblasts are trapped within an endomysial tube to form satellite cells, needed later for muscle growth and regeneration of damaged muscle fibres. There are many degenerative changes in foetal pig muscles, most likely from fibres that fail to anchor properly [4].

In the contemporary literature there are many exciting discoveries regarding muscle growth in pigs and the quality of the meat they produce - genes, biochemical pathways, too numerous to credit. But they must all work through Conrad Waddington's (1905 - 1975) epigenetic mechanisms, where the original definition of epigenetics being the causal analysis of development is most appropriate for use here [4]. And so it seems reasonable to return to classical discoveries and ignored stereological problems - what is the balance between hyperplasia (numbers of muscle fibres) and hypertrophy (growth of fibre diameters and lengths). Working through a 50-year personal data base on this topic, the idea was to follow MacCallum's stereology and to question the importance of tapered fibres in pig muscle growth.

\section{MATERIALS AND METHODS}

Muscle tissue from foetal pigs was fixed in $1 \%$ glutaraldehyde in $0.2 \mathrm{M}$ phosphate buffer at $\mathrm{pH} 7.4$ and embedded in Epon. Sections (1 to $3 \mu \mathrm{m}$ ) were cut with a glass knife and viewed by phase contrast microscopy. For histochemical characterization of post-natal muscle fibres, $10 \mu \mathrm{m}$ frozen sections were reacted for myosin adenosine triphosphatase (ATPase), succinate dehydrogenase (SDH), phosphorylase and periodic-acid Schiff for glycogen.

Quantitative histology of muscle growth is difficult because muscle fibres are contractile - when they contract they decrease in length and they increase in diameter. Thus, there may be a large error in one dimension if the other dimension is unknown or uncorrected. Also there are problems in obtaining muscle samples. Biopsy or immediate post-mortem samples exhibit a wide range in their contractile response to excision, but if muscles are left intact until rigor mortis occurs, muscle length may be affected by posture and muscle fibres may shrink as they leak fluid. Thus, accounting for the contributions of increasing muscle fibre numbers (hyperplasia) and increasing diameter and length of muscle fibres (hypertrophy) to growing muscle mass is complex. These problems were minimized as far as possible by tying excised 
muscle samples to small sticks at their original length before excision. Figure 3 shows how the diameters of myotubes and secondary fibres were measured.

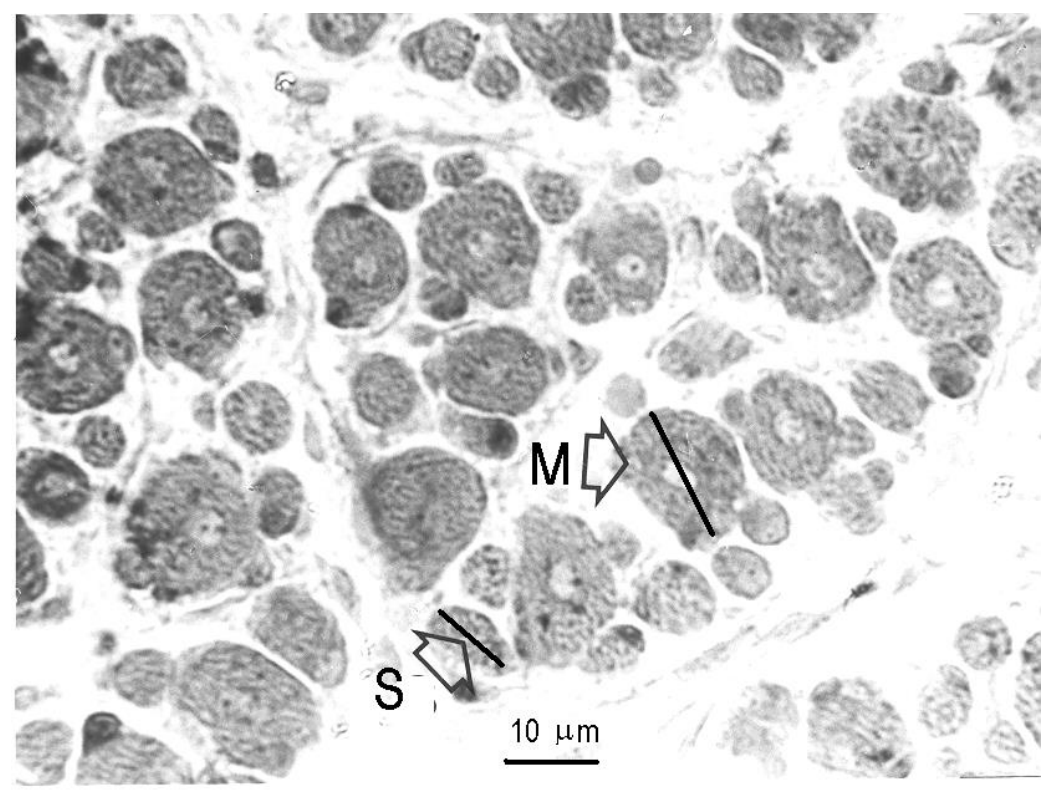

Fig. 3. Transverse section of Longissimus thoracis at 70 days gestation showing the method of measuring maximum diameters of myotubes $(\mathrm{M})$ and the axial myofibrillar cores of secondary fibres $(\mathrm{S})$.

\section{RESULTS}

\section{Pre-natal fibre diameters}

Secondary fibre diameters were compared in two muscles, Peroneus longus with short fasciculi lacking tapered fibres versus long fasciculi with tapered fibres of Longissimus thoracis (Fig. 4). Following MacCallum [2], the interpretation of the data was that Peroneus longus had few tapered fibres intruding into the plane of sectioning, while the longitudinal growth of the tapered fibres in Longissimus thoracis kept mean diameters low. In neither muscle was there any evidence of radial hypertrophy in secondary fibres - axial strands of myofibrils did not get thicker.

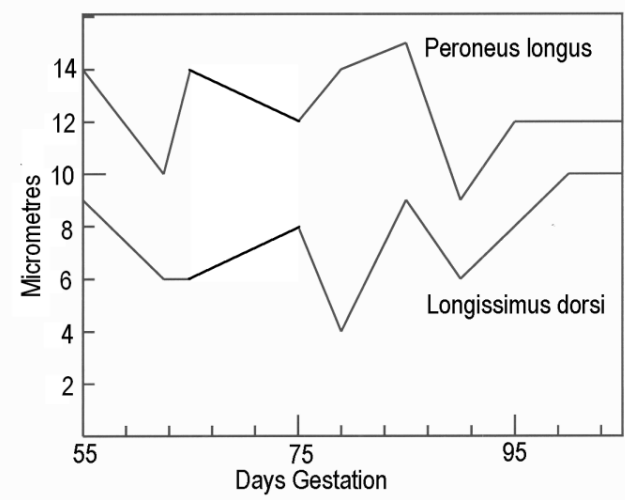

Fig. 4. Mean maximum diameters $(n=100)$ of the axial cores of myofibrils of secondary fibres in Peroneus longus and Longissimus thoracis muscles of foetal pigs. Differences were significant $P<0.001$ until 100 days and then $P<0.05$ [6].

In contrast to the lack of radial hypertrophy in secondary fibres (axial strands of secondary fibres did not grow thicker), there was a marked decrease in the diameters of myofibrillar tubes in myotubes $(r=-0.91, P<0.001)$ until around 105 days when the nuclei of myotubes had moved to a peripheral position so that there was little difference between fibres derived from myotubes and fibres derived from secondary fibres (Figure 5). The decrease in myotubes diameters was most likely caused by myotubes stretching and shedding the myoblasts and new secondary fibres on their surfaces. 


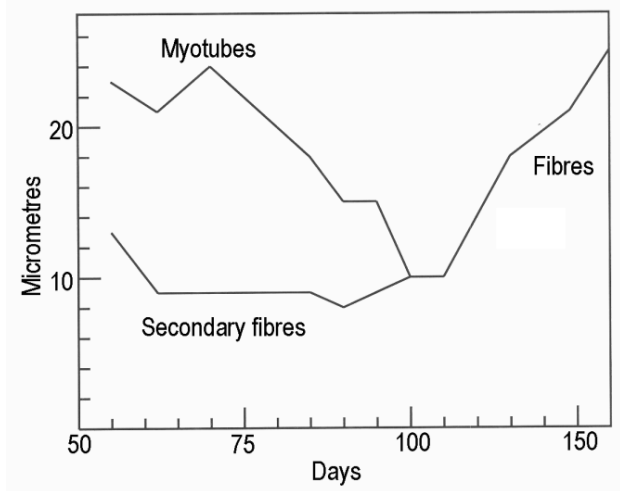

Fig. 5. Pooled data for mean diameters $(n=300)$ of the axial cores of myofibrils of secondary fibres, the diameters of myofibrillar tubes of myotubes, and maximum diameters of peri-natal muscle fibres in three muscles (Peroneus longus, Longissimus thoracis and Rectus femoris) of two breeds of pigs (Chester White and Poland China).

From Fig. 5 it is apparent that, until 100 days, developing muscles contained a mixture of myotubes and secondary fibres. Enumerating those with axial myofibrils (secondary fibres + peri-natal fibres) gave a useful estimate of the relative contributions of myotubes versus secondary fibres - approximately $80 \%$ of muscle fibres in pigs are derived from secondary fibres (Fig. 6).

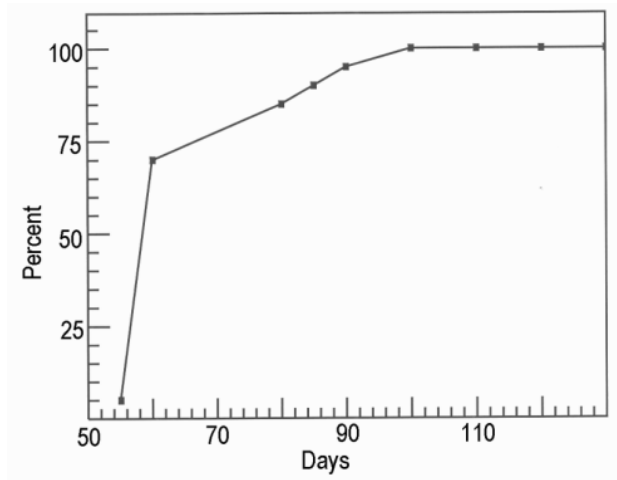

Fig. 6. The percentage of fibres having an axial core of myofibrils (secondary fibres plus all fibres after the disappearance of tubular structures) in the data of Fig. 5..

\section{Tapered fibres}

Schwann's [1] and MacCallum's [2] discoveries explain how new small diameter secondary fibres might grow in length to affect both mean fibre diameters and fibre numbers in a transverse section of a muscle. This also occurs during the later growth of pig muscles because of tapered fibres anchored in the endomysium of adjacent fibres. This was shown by dissection of post-natal porcine Sartorius muscles stained with silver nitrate and stored in glycerol. Fibres at approximately $100 \mu \mathrm{m}$ diameter were followed until they reached tapered endings (Figure 7). From MacCallum's stereology [2] , the longitudinal growth of tapered fibres at a faster rate than the length of their fasciculi may be able to increase the girth of a muscle [7].

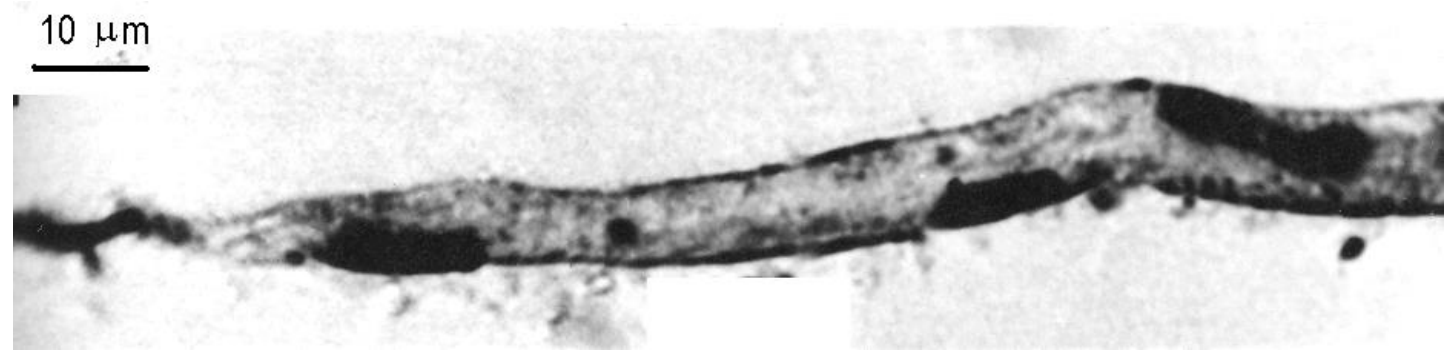

Fig. 7. A tapered fibre dissected from an adult Sartorius muscle [6,7]. 
The apparent number of muscle fibres at the midlength of the Sartorius, increased $(\mathrm{P}<0.005)$ through post-natal growth (Fig. 8) during the commercial growing period $(0.7 \mathrm{~kg}$ per day). When pigs were placed on a restricted diet at various times to stop growth in live weight, the increase in apparent fibre numbers stopped [8].

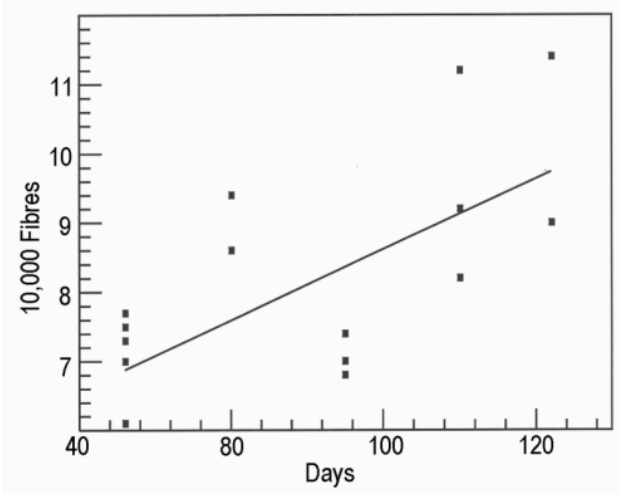

Fig. 8. Increase in the apparent number of muscle fibres at the midlength of the Sartorius muscles during the commercial growing period (days post-natal).

Tapered fibres easily escape detection in transverse sections unless the endomysium is stained with silver (Figure 9). Without this, tapered fibres appear as small angular parts of larger muscle fibres. That they are not parts of normal diameter fibres was further proved by looking for tapered fibres that differed histochemically from surrounding fibres. This also showed that endomysial staining is essential for the accurate measurement of muscle fibre diameters or cross sectional areas - without it the data area biased by missing all the small fibres and overestimating the size of larger fibres.

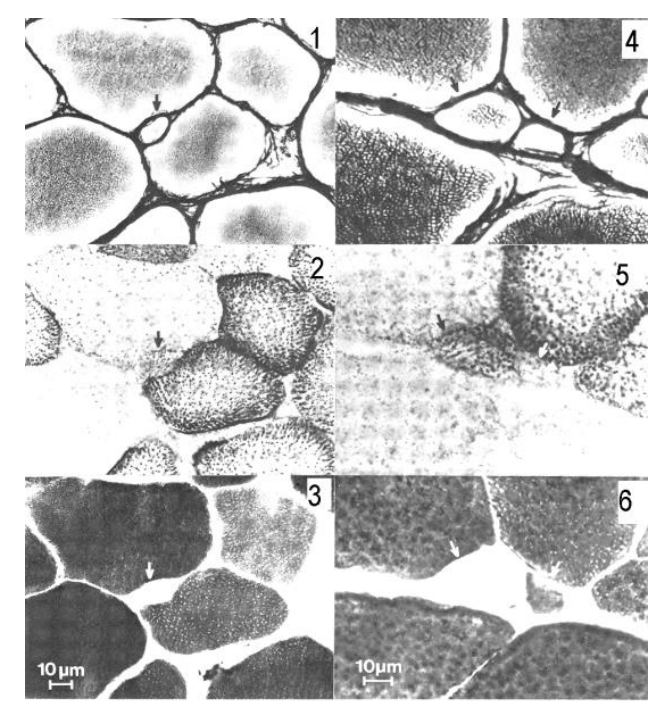

Fig. 9. Tapered fibres (shown by arrows) in serial sections of Biceps femoris with silver stained endomysium (1 and 4), succinate dehydrogenase (2 and 5) and alkali-stable myosin adenosine triphosphatase (3 and 6) [9].

\section{Longitudinal fascicular growth}

The practical importance of longitudinal fascicular growth was shown in a study of allometric growth in hind limb muscles of pigs [10]. Growth gradients in meat animals are extremely important commercially because the valuable meat cuts of the loin and proximal hind limb muscles have late developing high allometric growth rates, but this was not reflected in the growth of muscle fibre diameters nor in whole muscle cross-sectional areas. Explaining the late developing muscle mass in proximal hind limb muscles, whole muscles grew faster than bone lengths so that they bulged outwards, and proximal muscles had a faster rate of new sarcomere formation than distal muscles.

Another important commercial aspect of longitudinal fascicular growth concerns the cross sectional area of the Longissimus thoracis muscle seen in pork chops. As a butcher cuts pork chops perpendicularly to the vertebral column, the muscle fasciculi are cut at an angle so that muscle fibres have an elliptical cross section, although this is generally 
ignored by histologists who trim their tissue blocks to cut cross sections perpendicularly to the long axes of muscle fibres, thus leading to some erroneous conclusions about fibre diameters in relation to macroscopic muscle areas. Figure 10 shows this stereology for a typical pork chop. The depth of the muscle area (line A - B in Fig. 10) depends on the length of muscle fasciculi while only the width of the area (perpendicular to A - B) depends on the apparent number of fibres and their diameters [11].

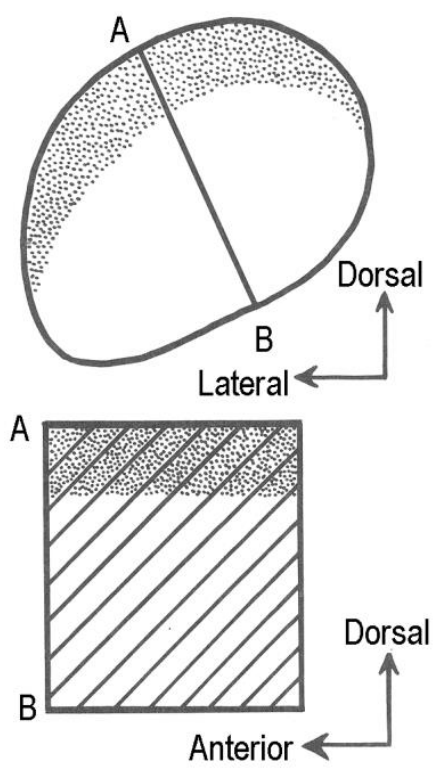

Fig. 10. The stereology of muscle fasciculi in the Longissimus thoracis of a pork chop, with the stippled area showing how growth in fascicular length contributes to muscle depth $(\mathrm{A}-\mathrm{B})$.

\section{Histochemical fibre types}

From Schwann [1] and Bardeen [4] we know that myotubes tend to be located centrally within fasciculi and that secondary fibres tend to be located peripherally in their fasciculi (Fig. 3). This mirrors the distribution of histochemical fibre types in the fasciculi of many porcine muscles (Fig. 11). Thus, myotubes in porcine muscle tend to form slow contracting fibres in the centre of their fasciculi, while secondary fibres tend to form fast contracting fibres on the outer parts of fasciculi. If there were no other epigenetic mechanisms involved, muscles would be locked into an approximate $20 \%$ to $80 \%$ ratio (myotubes versus secondary fibres as in Fig. 6), but this clearly is not the case. Soon after birth, histochemical fibre types start to change their characteristics [12] heading towards an appropriate balance for adult muscles. But how does this happen? Is this under the control of the motor innervation [13] or is it a result of differential growth of tapered fibres? Certain fibre types with tapered endings might have more rapid longitudinal growth than others. To answer this question, searches were made for one fibre type changing to another using microscope spectrophotometry. The result was the discovery of numerous transitional stages as one histochemical fibre type changed to another [14]. Microscope spectrophotometry also showed that most of the lactate formed during post-mortem glycolysis originated from fast contracting fibres in the periphery of their fasciculi [15]. Perhaps the differential longitudinal growth of fibres is involved, but there is no direct evidence, and neurally regulated epigenetics seems most likely at present. 


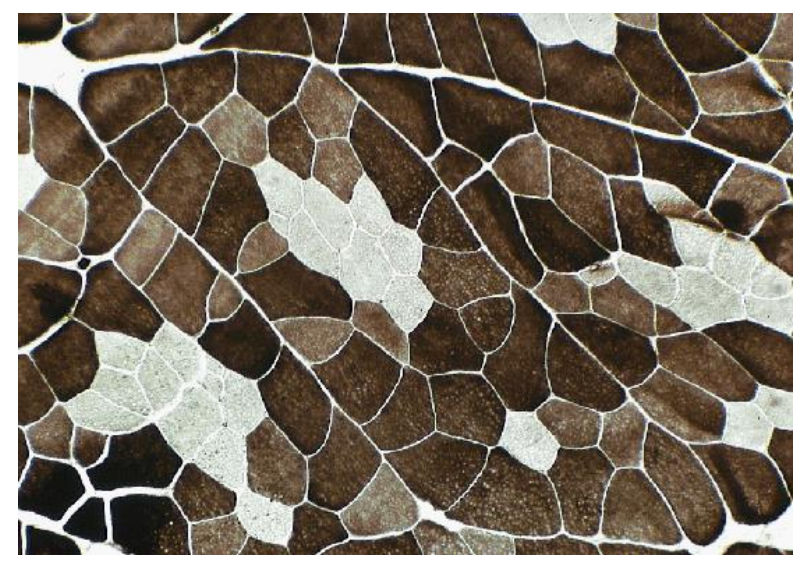

Fig. 11. The typical distribution of muscle fibres with alkali-stable myosin ATPase (fast contracting fibres ) in a pork muscle (12]). Note one tapered fibre on the left of the field.

\section{CONCLUSIONS}

These results conflict with researchers who think that muscle growth in pigs can be reduced to muscle fibre numbers and diameters and the genes that control them, and with those who think that pork quality is simply a question of histochemical fibre types. The evidence reviewed shows that the longitudinal growth of fibres is a key part of muscle development and that the nervous system also is involved. Not only does the nervous system modulate fibre histochemistry, but post-mortem events in the abattoir have a profound effect on muscle activation and lactate formation, the major determinants of pork quality [16]. Early post-mortem muscle activation lowers $p \mathrm{H}$ in a hot carcass, which has more effect than an eventual $p \mathrm{H}$ decline in a cold carcass [17].

To bring this retrospective review up to date, Fig. 12 shows the myoglobin concentrated in the centre of a pork muscle fasciculus. Meat colourimetry has recently advanced to the point that light scattering is now recognized as being a major factor in pork colour [18]. We can now see this originates mostly from the outer fibres of a fasciculus with a low myoglobin content (fast contracting fibres loaded with glycogen and responsive to early post-mortem activation). The centrally located fibres in pork fasciculi have a high mitochondrial content and mitochondria absorb light, so that light passing along the length of fibres is mostly in the peripheral fibres with a low myoglobin content [19]. Thus, not only do secondary fibres give rise to the majority of muscle fibres in pigs, but they also account for the transmittance and scattering of light which affects pork colour..

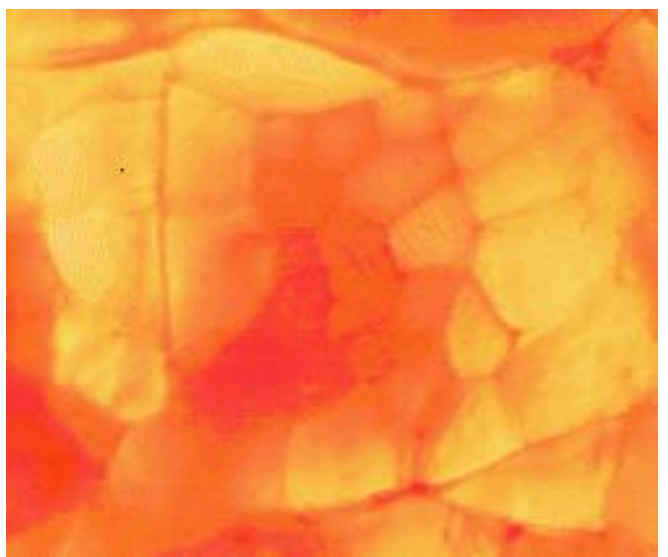

Fig. 12. The typical distribution of slow contracting muscle fibres with a high myoglobin content in the centre of a pork muscle fasciculus [11] 


\section{REFERENCES}

[1] Schwann, T. (1839) Mikroskopische Untersuchungen über die Uebereinstimmung in der Struktur und dem Wachsthum der Thiere und Pflanzen. Berlin: Verlag der Sander'schen Buchhandlung (G.E. Reimer), https://www.deutschestextarchiv.de/book/show/schwann_mikroskopische_1839

[2] MacCallum,J.B. (1898) On the histogenesis of the striated muscle fibre, and the growth of the human Sartorius muscle. Bulletin of the Hohns Hopkins Hospital. 208 9: https://babel.hathitrust.org/cgi/pt?id=coo.31924069247538\&view=1up\&seq=254\&skin=2021

[3] Waddington, C.H. (1952). The Epigenetics of Birds. Cambridge University Press. https://www.google.ca/books/edition/The_Epigenetics_of_Birds/HQs9AAAAIAAJ?hl=en\&gbpv=1\&dq=epigenetics +of+birds\&printsec $=$ frontcover

[4] Bardeen, C.R. (1900). The development of the musculature of the body wall in the pig, including its histogenesis and its relation to the myotomes and to the skeletal and nervous apparatus. Johns Hopkins Hospital Report. 9: 367-399. https://books.google.ca/books?id=PZE1AQAAMAAJ\&pg=PA141\&lpg=PA141\&dq=The+development+of+the+mu sculature+of+the+body+wall+in+the+pig,\&source=bl\&ots=10qiaGkbQQ\&sig=ACfU3U2sZmpv7CtkNPHs2jWMG AfPdebW9Q\&hl=en\&sa=X\&ved=2ahUKEwiQpN2O_NHzAhU1mGoFHUt9ARsQ6AF6BAgPEAM\#v=onepage\&q $=$ The $\% 20$ development $\% 20$ of $\% 20$ the $\% 20$ musculature $\% 20$ of $\% 20$ the $\% 20$ body $\% 20$ wall $\% 20$ in $\% 20$ the $\% 20$ pig $\% 2 \mathrm{C} \& \mathrm{f}$ $=$ false

[5] H.J. Swatland and R.G. Cassens, (1973). Pre-natal development, histochemistry and innervation of porcine muscle. Journal of Animal Science 36: 343-354. DOI 10.2527/jas1973.362343x

[6] Swatland, H.J. (1971). Development and Innervation of Muscle Subject to Selective Breeding. Ph.D. Thesis, University of

Wisconsin https://books.google.ca/books/about/Development_and_Innervation_of_Muscle_Su.html?id=bHbUAAAAMAAJ\&r edir_esc $=$ y

[7] Swatland, H.J. and Cassens, R.G. (1972). Muscle growth: the problem of muscle fibers with an intrafascicular termination. Journal of Animal Science 336-344. https://www.researchgate.net/publication/19049265_Muscle_Growth_The_Problem_of_Muscle_Fibers_with_an_Int rafascicular_Termination\#fullTextFileContent

[8] Swatland, H.J. (1976). Effect of growth and plane of nutrition on apparent muscle fibers numbers in the pig. Growth 40:

285-292.

https://www.researchgate.net/publication/22177734_Effect_of_growth_and_plane_of_nutrition_on_apparent_muscl e_fiber_numbers_in_the_pig

[9] Swatland, H.J. (1983). The histochemistry of very small muscle fibres in growing skeletal muscles. Journal of the Neurological Sciences 61: 435-442. DOI: 10.1016/0022-510X(83)90176-4

[10] Swatland, H.J. (1982). Histology of allometric growth in hindlimb muscles of pigs. Journal of Agricultural Science, Cambridge 98: 629-632. DOI: 10.1017/S0021859600054423

[11] H.J. Swatland, (2006). Growth and Structure of Meat Animals. An undergraduate teaching site with original micrographs by the author. https://animalbiosciences.uoguelph.ca/ swatland/gasman.html

[12] Swatland, H.J. (1975) Histochemical development of myofibers in neonatal piglets. Research in Veterinary Science 18: 253-257. DOI: 10.1111/j.1439-0442.1977.tb01570.x

[13] Sakuma, K. and Yamaguchi, A. (2011). The recent understanding of the neurotrophin's role in skeletal muscle adaptation. Journal of Biomedicine and Biotechnology . doi: 10.1155/2011/201696. Epub 2011 Sep 25. 
[14] Swatland, H.J. (1977). Transitional stages in the histochemical development of muscle fibres during post-natal growth. Histochemical Journal 9: 751-7. DOI: 10.1007/BF01003069

[15] Swatland, H.J. (1977). Cytophotometry of postmortem glycogenolysis in different histochemical types of muscle fibres in the pig. Histochemical Journal 9: 163-170. DOI: 10.1007/BF01003628

[16] Swatland, H.J. (1983). Measurement of electrical stunning, rate of exsanguination, and reflex activity of pigs in an abattoir. Canadian Institute of Food Science and Technology 16: 35-38. DOI: 10.1016/S0315-5463(83)72016-X

[17] Bendall, J.R and Swatland, H.J. (1988). A review of the relationships between $\mathrm{pH}$ and physical aspects of pork quality. Meat Science 24: 85-126. DOI: 10.1016/0309-1740(88)90052-6

[18] Swatland, H.J. (2021). An explanation of subsurface optical pathways through food myosystems. Asian Journal of Agriculture and Food Sciences 9: 143-157. DOI: 10.24203/ajafs.v9i4.6706

[19] Swatland, H.J. (2004). Absorbance of light by mitochondria in pork muscle fibres differing in myoglobin content. Meat Science 67: 371-375. DOI: .org/10.1016/j.meatsci.2003.11.005 